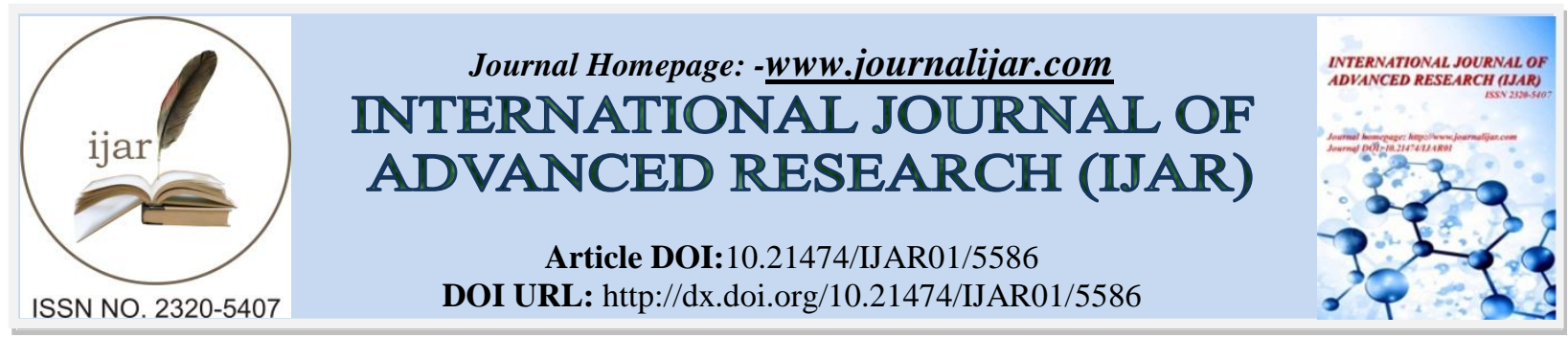

RESEARCH ARTICLE

\title{
OUTCOMES OF INDUCED MEDICAL ABORTION IN FIRST TRIMESTER OF PREGNANCY: REPORT FROM A TERTIARY CARE HOSPITAL
}

Dr. Sasmita Swain and Dr.Sujata Singh.

Department of Obstetrics and Gynecology, S.C.B medical College and Hospital, Cuttack, Odisha, India.

\section{Manuscript Info}

Manuscript History

Received: 10 August 2017

Final Accepted: 12 September 2017

Published: October 2017

\section{Abstract}

Background:Medical abortion is a safe, effective and affordable method of termination of pregnancy. Success rate is $92-97 \%$. When performed as per guidelines. After legalisation of medical abortion in India in 2002, abortion pills are available over the counter throughout the country. This has led to unsupervised use of such method and subsequent complications.

Objective:To study the demographic profile and outcomes of induced medical abortion in first trimester of pregnancy.

Materials and methods:This is a hospital based prospective observational study undertaken in the department of Obstetrics \&Gynaecology, S.C.B. Medical College, Cuttack during the period of one year from August 2015 to July 2016.

Women between 15 to 45 years in first trimester of pregnancy admitted the department either through emergency or OPD after taking combined abortificient drugs (mifepristone \& misoprostol combinations) elsewhere were included in the study.

Results:Out of 123 cases $91.8 \%$ were married, $61.8 \%$ were from early reproductive age group $74.4 \%$ (20 to 30 years) are from rural area and $78.9 \%$ belongs to low socio-economic section. $91.1 \%$ women taken these drugs either through over the counter or from huregistences medical practitioner. $78.9 \%$ of women taken the drug within 9 weeks gestation (63 days). $88.6 \%$ of cases presented to the hospital with excessive vaginal bleeding and $43.9 \%$ with abdominal pain. Incomplete, complete and septic abortion was detected in 73.9\%, 10.6 and 12.2 percent of cases respectively. $38.2 \%$ were detected to have severe anaemia and $57.7 \%$ moderate anemia, $47.2 \%$ needed blood transfusion, 3 (2.4\%) patients expired due to anemia, sepsis, shock and ARF.

Conclusions:Most of the women are young, less educated and low socio-economic status. Incomplete abortion, bleeding PV with anemia and infection were common outcomes. Large prospective studies are required to assess the magnitude of the problem.

Copy Right, IJAR, 2017,. All rights reserved.

Corresponding Author:- Dr Sujata Singh. 


\section{Introduction:-}

Induced abortion safe or unsafe, legal or illegal is a health problem in the reproductive age of woman in high developed and developing countries. More than one third of the approximate 205 million pregnancies that occur each year around the world are unplanned and about $20 \%$ of them end in induced abortions(1). Most of these abortions take place during the first trimester of pregnancy, only 10 to $15 \%$ occur during second trimester. In India the majority of abortions are either not reported or take place illegally. As reported in Family Welfare stastician in 2011, the number of induced abortions in India in approved centres is sestimated to be 6,20,472 and the maternal mortality due to unsafe abortion is 8\%(2). Medical abortion is legalised in India since 2002. (Ammendment of MTP Act 2002) Medical abortion using a combination of drug (mifepristone and misoprostol) is a safe, efficient, affordable, acceptable and approved method of MTP upto 9 weeks (63 days) of gestation(3). Due to easy availability of the drugs over the counter (OTC) many take these drugs themselves without seeking proper medical advise or with unauthentic prescriptions from unregistered medical practitioners. Incomplete abortion anemia and sepsis are known complication of medical abortion. These problems become worse if there is improper selection patient drug dose, route and timing of administration. This study was undertaken to observe the outcomes after undergoing medical abortion in first trimester of pregnancy admitted to our institution.

\section{Materials and methods:-}

This is a hospital based observational study with reference to outcomes and complications of medical methods of abortion in first trimester of pregnancy, carried out in the Department of Obstetrics \&Gynaecology, S.C.B. Medical College, Cuttack during the period of one year from August 2015 to July 2016.

Only the cases admitted in our department either through Labour room (emergency) or O.P.D after taking combined abortifacient drugs (mifepristone \& misoprostol combinations) elsewhere were taken in my study.

\section{Inclusion Criteria:-}

Women who had undergone medical methods of abortion using mifepristone \& misoprostol combined drugs after confirming pregnancy by urine pregnancy test.

Age of patient should be between 15 to 45 years

Period of gestation $<12$ weeks

The patient should be enrolled after her informed consent to participate in the study and follow up.

\section{Exclusion Criteria:-}

Women who had undergone first trimester MTP with surgical methods

Period of gestation $>12$ weeks

Those refused for consent

Ethical clearance was obtained from institutional ethics committee.

\section{Procedure:-}

Complete recording was made regarding the age of patient, parity, marital status, socio-economic status, duration of gestation, methods of interference, type \& dose of drug used, legal or illegal use \& the presenting features.

A through clinical examination of other systems including pelvic examination was done in all cases.

Women with history of use of medical abortion pills were asked in details about methods used;

1. Regarding source of medication

2. Regarding proper prescription given or not

3. Onset of bleeding \& its severity

4. Interval between the onset $\&$ reporting to hospital

5. Treatment received before referral

6. Advice regarding proper follow up etc.

During the preliminary steps internal pelvic examination done, wherever needed pregnancy was confirmed either by urine pregnancy test or further collaborated by ultrasonography.

In this study WO recommendation for first trimester medical abortion was taken as standard and according compliance and outcomes were studied. 
Investigations- apart from routine investigations like $\mathrm{Hb} \%, \mathrm{PCV}, \mathrm{DC} \mathrm{TLC}$, Blood grouping \& Rh typing, urine routine \& microscopic examination, infectious screening and ultrasonography for all cases.

Some relevant investigations like BT, CT, TPC, RFT, LFT, Serum electrolytes, MP ICT, HVS C \& S, Blood Culture were done for selective cases.

All the data was entered in Microsoft ecel and analysed using SPSS 21.0 software. Statistical data were analysed with the chi square test and multivariate analysis using binary logistic regression model.

Standard management was done according to patients clinical condition whenever required blood transfusion was given and curettage was done.

\section{Results:-}

The total no. of induced abortions admitted during the study period was $123.94(74.4 \%)$ patients were from rural area, 97 (78.9\%) from low socio-economic class and $113(91.8 \%)$ were married. Most patients were house wife (111 - 90.2\%) and multigravida $40(32.5 \%)$ patients were above 30 years and $83(67.5 \%)$ were within thirty years of age (Table 1). Maximum induced abortions were in 7 - 9 gestational weeks $(58-47.2 \%)$, followed by gestational age $<7$ weeks $(39-31.7 \%)$ and $9-12$ weeks $(26-21.1 \%)$ Table 2, $75(61 \%)$ patients have procured drugs directly from medicine counter, $37(30.1 \%)$ from unregistered and only $11(8.9 \%)$ from registered medical practitioners. It was observed that $52(42.3 \%)$ patients taken the drugs as per WHO recommendation. Bleeding PV was noticed by the woman in less than $24 \mathrm{hr}$ after pill intake in 13 (10.6\%), 24 to 48 hours in $10(82.1 \%)$ and beyond 48 hours in 9 (7.3\%) patients (Table 3). Pill intake and hospital visit interval was 1 - 3 days in 15 (2.2\%) and beyond 14 days in 15 $(12.2 \%)$ patients (Table 4). Excessive bleeding per vagina nor the most common presenting complaints (88.6\% and 109 patients). Second most common symptom was abdominal pain (43.9\%, 54 patients). Table 5 after common complaints were fever and fowl smelling discharge (12.2\% each). Some patients were admitted with more than one complaints. Outcome following abortion pills in this study is shown in table - 6. Incomplete abortion (739.\%) were most common followed by septic abortion (12.2\%). Mild to moderate anemia was detected in $71(57.7 \%)$ and severe anemia in $47(38.2 \%)$ patients. (Table - 7) Management of these patients has been shown in table - 8. Maximum women were managed with suction and evacuation (S\&E) with antibiotics (106 [86.2\%)]. 3 patients under went emergency laparotomy for ruptured ectopic pregnancy. Out of all 58 patients required blood transfusion. Duration of hospitalisation was upto $24 \mathrm{hrs}$ in $34(22.6 \%), 1$ to 3 days in $63(51.2 \%), 3$ to 5 days in $10(8.1 \%)$ and more 8 hrs 5 days in $16(13.1 \%)$ patients, $104(84.5 \%)$ patients were discharged after proper medical care, 15 stable cases left against medical advice ane one continued her pregnancy. 3 patients expired, all with bleeding, anemia and shock.

Multivariate analysis using binary logistic regression model, taking gestational age as dependant variable detected the following:

1. The chance of incomplete abortion was more if drug schedule was not proper and not advised by RMP.

2. The requirement of blood transfusion were more in women who had taken ahortificient drug after 9 weeks of gestation (p-0.0001).

3. Septic abortion was more common when drugs taken after 9 weeks of gestation (P-0.0008).

4. Shock more common after 9 weeks gestation $(\mathrm{P}<0.01)$.

Table 1:-Age Distribution

\begin{tabular}{|c|c|c|}
\hline AGE & NUMBER & \% \\
\hline$<20$ YRS & 7 & 5.7 \\
\hline $21-25$ YRS & 22 & 17.9 \\
\hline $26-30$ YRS & 54 & 43.9 \\
\hline$>30$ YRS & 40 & 32.5 \\
\hline
\end{tabular}

The incidence were more in age groups 26-30 yrsi.e 54 (43.9\%), next to between age groups $>30$ yrsi.e 40 (32.55) followed by between age group 21 - 25 yrsi.e 22 (17.9\%) and less in age groups <20 yrsi.e 7 (5.7\%). Overall incidence was more in age group 21-30 years 76 (61.8\%), representing reproductive group.

Table 2:-Gestational Age Distribution

\begin{tabular}{|c|c|c|}
\hline GESTATIONAL AGE & NUMBER & \% \\
\hline <OR $=$ 7 WEEK & 39 & 31.7 \\
\hline 7-9 WEEK & 58 & 47.2 \\
\hline
\end{tabular}




\section{\begin{tabular}{l|r|r} 
9-12 WEEK & 26 & 21.1
\end{tabular}}

Maximum induced abortion were done in 7-9 gestational weeks 58 (47.2\%), followed by in gestational age <7 weeks $39(31.7 \%) \& 26(21.1 \%)$ were induced after 9 weeks of pregnancy.

Table 3:-Pill Intake - Abortion Interval

\begin{tabular}{|c|c|c|}
\hline PILL INTAKE-ABORTION INTERVAL & NUMBER & \% \\
\hline$<24$ HOURS & 13 & 10.6 \\
\hline $24-48$ HOURS & 101 & 82.1 \\
\hline$>48$ HOURS & 9 & 7.3 \\
\hline
\end{tabular}

$101(82.1 \%)$ were noticed the onset of bleeding (process of abortion) within 48 hours. 13 (10.6\%) women starts bleeding within 24 hrs\& $9(7.3 \%)$ women after 48 hrs.

Table 4:- Pill Intake-Visit To Hospital Interval

\begin{tabular}{|c|c|c|}
\hline PILL INTAKE-VISIT TO HOSPITAL & NUMBER & \% \\
\hline 1-3 DAYS & 93 & 75.6 \\
\hline 4-7 DAYS & 8 & 6.5 \\
\hline 8-14 DAYS & 7 & 5.7 \\
\hline$>14$ DAYS & 15 & 12.2 \\
\hline
\end{tabular}

Out of 123 cases $93(75.6 \%)$ were admitted within 3 days of pill intake, followed by 15 (12.2\%) were within 2 weeks and another 15 (12.2\%) were admitted after 14 days.

Table 5:-Presentation To The Hospital

\begin{tabular}{|c|c|c|}
\hline COMPLAINTS TO THE HOSPITAL & NUMBER & $\%$ \\
\hline EXCESSIVE BLEEDING PER VAGINA & 109 & 88.6 \\
\hline FOUL SMELL & 15 & 12.2 \\
\hline ABDOMINAL PAIN & 54 & 43.9 \\
\hline FEVER & 15 & 12.2 \\
\hline NO EXPELLED PRODUCT & 15 & 12.2 \\
\hline
\end{tabular}

$109(88.6 \%)$ cases were admitted with chief complaints of excessive bleeding per vaginum, 54 (43.9\%) were with abdominal pain, and $15(12.2 \%)$ were admitted with features of septic abortion. There are some patients who were admitted with more than one symptoms.

Table 6:-Type Of Abortion

\begin{tabular}{|c|c|c|}
\hline OUTCOME FOLLOWING ABORTION PILLS & NUMBER & $\%$ \\
\hline INCOMPLETE & 91 & 73.9 \\
\hline COMPLETE & 13 & 10.6 \\
\hline SEPTIC & 15 & 12.2 \\
\hline ECTOPIC & 3 & 2.4 \\
\hline MOLAR & 1 & 0.9 \\
\hline
\end{tabular}

After taking combined abortion pills $91(73.9 \%)$ were admitted with incomplete abortion and 15 (12.2\%) were with features of septic abortion and only $13(10.6 \%)$ were admitted with bleeding per vagina, later confirmed as complete abortion by ultrasound. $3(2.4 \%)$ cases were detected as ectopic pregnancy and 1 case as molar pregnancy.

Table 7:-Hb Level

\begin{tabular}{|c|c|c|}
\hline Hb level (gm\%) & NUMBER & \% \\
\hline$<7$ & 47 & 38.2 \\
\hline 7 to 10 & 71 & 57.7 \\
\hline$>10$ & 5 & 4.1 \\
\hline
\end{tabular}

Out of 123 cases admitted $47(38.2 \%)$ were detected as severe anemia. $71(57.7 \%)$ were having moderate anemia and only $5(4.1 \%)$ were having haemoglobin level more than $10 \mathrm{gm} \%$.

Table 8:- Management

\begin{tabular}{|c|c|c|}
\hline MANAGEMENT & NUMBER & \% \\
\hline S \& E & 51 & 41.4 \\
\hline
\end{tabular}




\begin{tabular}{|c|c|c|}
\hline S \& E WITH BT & 55 & 44.8 \\
\hline CONSERVATIVE & 14 & 11.4 \\
\hline LAPAROTOMY & 3 & 2.4 \\
\hline
\end{tabular}

Maximum women were managed with S\&E with antibiotics $106(86.2 \%)$ and out of them more than half 55 (44.8\%) required blood transfusion. Only $14(11.4 \%)$ cases managed conservatively as cases detected as complete abortion 3 $(2.4 \%)$ women undergone emergent laparotomy as diagnosed ruptured ectopic pregnancy.

\section{Discussion:-}

Unsafe abortion is a preventable cause of maternal mortality and morbidities. The MTP act of India legalized abortion with the aim of reducing maternal deaths due to unsafe abortions. But still $8 \%$ of maternal deaths are attributed to unsafe abortions in India(2). 5 million unsafe abortions are performed per year in India. To make medical abortion safe on laid down in comprehensive abortion care training and service guidelines include counselling, willingness for three visits, readiness for surgical method in case of failure, additional consent and MMA medical method of abortion client card with details of the patient along with details of doctor and place to report along with contact number in case of emergency(4). When guidelines azre followed meticulous failure rate is low and complications are few.

In this study $91.1 \%$ of women rasing procured the drug without the prescription of registered medical practitioner. In most of the cases prior examination not done, pregnancy not confirmed, and drug administration not supervised and there was no adherence to the recommended schedule. Similar results were reported in few recent studies $(5,6,7)$, $42.3 \%$ of women consumed fill drug dosage and $57.7 \%$ had not taken all the drugs due to ignorance and lack of counselling.

$76.4 \%$ of patients belong to rural area, $78.9 \%$ from low socioeconomic status, $91.8 \%$ married, $5.7 \%$ nonmarried and $2.5 \%$ were widows. Most are multigravida and above 25 years of age $(76.4 \%)$. This result are in accordance with other recently reported series $(8,9)$. Most women used this method of abortion to limit their family size and few to get rid of undesired pregnancy. In our study $31.7 \%$ of cases taken ahortificient drugs with in 7 weeks of gestation, $47.2 \%$ between 7 to 9 weeks of gestation and $21.1 \% 9$ to 12 weeks of gestation similar to other reports $(6,8,10)$. Pill intake to abortion interval was 24 to 48 hours in $82.1 \%$ of cases, $10.7 \%$ within 24 hours and $7.3 \%$ beyond 48 hours $75.6 \%$ were admitted to hospital within 3 days of taking drugs, $12.2 \% 4$ to 14 days and another $12.2 \%$ beyond 14 days. This is because many women had not taken the misoprostal and other taken not in appropriate regimen due to lack of preabortioncounselling. This is similar to that of K Nivedita et al (8).

Many patients had multiple symptoms. Most common presenting complaint was excessive bleeding per vagina (88.6\%) followed by abdominal pain $(43.9 \%)$ and found smelling discharge (12.2\%) and fever (12.2\%), which is similar to other reported recently $(5,8,9) 73.9 \%$ of our patients had incomplete abortion followed by $12.2 \%$ septic and $10.6 \%$ complete abortion. On ultrasonography 3 patients detected to have ectopic pregnancy and one molar pregnancy. Incomplete abortion was $62.5 \%$ in K. Nivedita $79 \%$ in NeelamGoyal and $83.3 \%$ in SikhaVerma series $(6,8,9) .38 .2 \%$ of cases had severe anemia $\mathrm{Hb} \%<7 \mathrm{gm} \%)$ and $57.1 \%$ had mild to moderate anemia ( $\mathrm{Hb} 7 \mathrm{to} 10$ gm\%) $5(4.1 \%)$ of out of RP patients presented with hemorrhagic shock. It was most common complication seen after medical abortion pills. In India many women in their reproductive age group have much to moderate anemia. Excessive bleeding PV further decrease after hemogrobin level.

Of the 123 patients in our series 51 (41.4\%) were managed with S \& E only 55 (44.8\%) needed blood transfusion along with S\&E, 14 (11.4\%) managed conservatively. All 3 (2.4\%) patients with ectopic pregnancy underwent emergency laparotomy, needed blood transfusion and survived, 3 (2.4\%) patients expired (maternal death), two primarily due to excessive bleeding and one due to sepsis leading to shock, ARF and death. Blood transfusion was required in $58(47.2 \%)$ of cases in our series. Pre-existing anaemia, unawareness of amount of blood loss and delayed hospitalisation leading to severe anaemia the cause of blood transfusion in most cases. Blood transfusion rate in other reported series vary greatly. In one study by Padwa et al 2016, 63\% needed blood transfusion and in another study by Sarojini et al 75\% need blood transfusion. In most other series rate of blood transfusion is low.

It was observed by multivariate analysis using binary logistic regression model that incomplete abortion, need for blood transfusion, septic abortion and shock are significantly more common in women with medical abortion in 9 12 weeks of gestation compared to that of less than 9 weeks of gestation.

Conclusion 
Complication and poor outcome following induced medical abortion is mainly due to ignorance, lack of medical facilities and purposeful avoidance trained personnel in cause of women to maintain social secrecy. Awareness regarding use of abortificient drugs, its dosage, method of use and follow up counselling in essential for the user and drug provider. Strang steps to be taken against misuse of such changes by untrained providers, pharmacists and patients themselves. 